\title{
Effet des glucocorticoïdes sur la capacité de dilution du rein chez un rongeur désertique : Gerbillus campestris
}

\author{
MA Laamarti 1, K Baddouri 1, C Burlet 2 \\ 1 Faculté des sciences, laboratoire de physiologie animale, unité d'endocrinologie, Rabat, Maroc; \\ ${ }^{2}$ CHU Brabois, Laboratoire d'exploration fonctionnelle rénale et métabolique, Nancy, France
}

(Reçu le 19 juin 1990; accepté le 24 avril 1991)

\begin{abstract}
Résumé - La capacité d'excrétion d'une surcharge hydrique égale à $5 \%$ du poids corporel a été étudiée chez le rat wistar et chez la gerbille (Gerbillus campestris). Le rat excrète la totalité de la surcharge en moins de $2 \mathrm{~h}$, alors que la gerbille excrète moins de $60 \%$ en $4 \mathrm{~h}$. Les gerbilles qui ont reçu une dose de $15 \mu \mathrm{g} / 100 \mathrm{~g}$ de poids corporel de dexaméthasone améliorent leur taux d'excrétion qui peut atteindre en moyenne $92 \pm 6 \%$ en 2 h 30 min. L'hormone antidiurétique (ADH) mesurée par radio-immunologie au moment du maximum de diurèse est indétectable chez les rats; par contre, chez les gerbilles, les taux d'ADH restent relativement élevés $(55 \pm 6,7 \mathrm{pg} / \mathrm{ml})$. L'incapacité partielle du rein de la gerbille à excréter une surcharge hydrique semble donc due au taux élevé d'ADH plasmatique et à une faible concentration en gluco-corticoïdes.
\end{abstract}

rongeur désertique / rein / excrétion hydrique / ADH / glucocorticoïde / dilution de l'urine

Summary - Effect of glucocorticoids on renal diluting capacity in a desert rodent: Gerbillus campestris. The ability to excrete a water load was studied in Wistar rats and in gerbils (Gerbillus campestris). The rat excreted the entire water load in less than $2 h$ whereas Gerbillus campestris excreted less than $60 \%$ of the water load in $4 \mathrm{~h}$. The gerbils which had received a dose of $15 \mu \mathrm{g} / 100 \mathrm{~g}$ body weight dexamethasone improved their rate of excretion which attained $92 \pm 6 \%$ in $2 \mathrm{~h} 30 \mathrm{~min}$. The andidiuretic hormone $(A D H)$ measured by radioimmunoassay at the time of maximum diuresis was undetectable in rats; in contrast, in gerbils the level of $A D H$ remained relatively high $(55.4 \pm 6.7$ $\mathrm{pg} / \mathrm{m} /$ ). We conclude that the partial inability of the gerbil's kidney to excrete a water load is due to a high ADH level and probably to a low concentration of glucocorticoids.

desert rodent / kidney / water excretion / ADH / gluco-corticoid / urinary dilution 


\section{INTRODUCTION}

Certains rongeurs désertiques sont caractérisés par une faible capacité de dilution du rein. À la suite d'une surcharge hydrique (5\% du poids coporel), Dipodomys merioni (Hofman, 1956) excrète uniquement $40 \%$ de cette surcharge en $6 \mathrm{~h}$ et Jaculus orientalis élimine dans le meilleur des cas $60 \%$ au plus en $5 \mathrm{~h}$ (Baddouri et al, 1987), alors que chez la gerbille ( $\mathrm{Me}$ riones shawii) en régime alimentaire dépourvu d'eau (Hummel, 1963), la charge d'eau intragastrique n'induit pas de diurèse aqueuse. Pour certains auteurs, cette altération de la capacité de dilution du rein serait due à une hyperproduction d'hormone antidiurétique (ADH) par le système hypothalamoneurohypophysaire (Ahmed et al, 1967; Boykin et al, 1978; Mandel et al, 1980 ) et/ou à une déficience en glucocorticoïdes (Kleeman et al, 1964; Green et al, 1970; Linas et al, 1980). La capacité de dilution du rein et l'effet d'un glucocorticoïde de synthèse (dexamthasone) sur le pouvoir d'excrétion d'eau par le rein ont été étudiés chez Gerbillus campestris (famille des Gerbillidés), rongeur caractérisé par une large répartition dans les zones arides et semi-arides (Petter, 1961).

\section{MATÉRIEL ET MÉTHODES}

L'expérimentation a été réalisée comparativement chez des rats et des gerbilles. Les rats étaient nourris de granulés (Cicalim) et d'orge, avec libre accès à l'eau, et les gerbilles ont été adaptées pendant une durée de 4 sem à recevoir un régime alimentaire riche en eau, composé d'orge et de $5 \mathrm{~g}$ de feuilles de laitue par jour et par animal. Avant l'expérimentation proprement dite, les animaux (non anesthésiés) ont été entraînés pendant 5 jà recevoir des volumes croissants d'eau par tubage intragastrique. Après un jeûne de $12 \mathrm{~h}$, dans une première série d'expériences, un premier lot d'animaux composé de rats et de gerbilles a reçu une quantité d'eau équivalente à $5 \%$ de leur poids corporel, puis les animaux furent placés en cage à métabolisme. À chaque miction, le temps fut noté et le volume urinaire déterminé par pesée. Dans une deuxième série expérimentale, l'effet de la surcharge hydrique sur l'ADH circulante a été recherché chez les mêmes animaux qui ont été sacrifiés au moment du maximum de la diurèse, c'est-à-dire lorsque les urines ont l'osmolalité la plus basse. Dans les plasmas, l'ADH, la natrémie et l'osmolalité furent mesurés et comparés à des animaux non hydratés (deuxième lot de gerbilles). L'effet de la dexaméthasone sur l'excrétion de la charge d'eau a été suivi sur un troisième lot de gerbilles, qui ont reçu au moment du gavage, par injection intrapéritonéale, une dose de 15 $\mu \mathrm{g} / 100 \mathrm{~g}$ de poids corporel. Chez les mêmes animaux, la cinétique d'excrétion de la surcharge fut d'abord mesurée en l'absence de dexaméthasone (injection de sérum physiologique).

L'ADH plasmatique fut extraite sur des colonnes Octyl-Silice $500 \mathrm{mg}$ (Amersham) après ajustement à $\mathrm{pH} 3$ des échantillons avec $\mathrm{HCl}$ $2 \mathrm{~mol}^{-1} \mathrm{I}^{-1}$. La silice était ensuite lavée avec $15 \mathrm{ml}$ d'acide trifluoroacétique à $0,1 \%$ et l'ADH éluée avec $2 \mathrm{ml}$ d'un mélange acétonitriletrifluoroacétique à $0,1 \%$. Les éluats furent évaporés à sec à $37^{\circ} \mathrm{C}$ sous azote et l'extrait sec fut repris avec $250 \mu \mathrm{l}$ de tampon phosphate 0,01 mol. I-1 $^{-1} \mathrm{pH} 7,6$. L'ADH a été ensuite dosée par radio-immunologie (Klein et al, 1966; Marchetti et al, 1973). L'immunsérum permet de détecter $1 \mathrm{pg}$ d'hormone, et $17 \mathrm{pg}$ d'AVP déplacent $50 \%$ de l'hormone marquée, liée à l'anticorps.

L'osmolalité plasmatique et urinaire a été mesurée par cryométrie (Osmomètre Knauer). À chaque miction, les volumes urinaires excrétés ont été déterminés par pesée et exprimés en pourcentage du volume d'eau administré. La clairance d'eau libre $\left(C_{\mathrm{H}_{2} \mathrm{O}}\right)$ a été calculée au maximum de la diurèse selon la formule $C_{\mathrm{H}_{2} \mathrm{O}}=$ $\dot{V}\left(1-U_{o s m} / P_{o s m}\right), \dot{V}$ étant le débit urinaire $(\mathrm{ml} /$ $\mathrm{min}$ ). La concentration du sodium a été mesurée par photométrie de flamme. Le délai d'installation de la diurèse séparant la surcharge hydrique du début de la formation de l'urine est obtenu par extrapolation de la courbe sur l'axe des abscisses. Les résultats sont exprimés en moyenne \pm ESM (erreur standard à la moyenne). 
La validité statistique des différences entre les moyennes de 2 séries expérimentales est évaluée d'après le test de Fisher-Student. La différence entre les moyennes est considérée statistiquement significative au seuil de $5 \%(P<$ $0,05)$.

\section{RÉSULTATS}

\section{Cinétique d'excrétion urinaire}

Les gerbilles dont le poids varie entre 22 et $25 \mathrm{~g}$ ont reçu une surcharge hydrique comprise entre 1,1 et 1,25 ml. La charge d'eau reçue par les rats était environ 6 fois plus élevée puisque leur poids variait entre 120 et $150 \mathrm{~g}$. La cinétique de l'excrétion urinaire a été mesurée sur une durée expérimentale de $7 \mathrm{~h}$ environ (fig 1 ) : alors que le rat élimine la totalité de la surcharge en 92 $\pm 5 \mathrm{~min}$, les gerbilles, après avoir éliminé environ la moitié de la surcharge $(57,5 \pm$ $2,5 \%$ en $218 \pm 9 \mathrm{~min}$ en moyenne), ont une diurèse extrêmement ralentie (fig 1). Le délai d'installation de la diurèse est beaucoup plus long chez la gerbille $(67,1 \pm$ $3,6 \mathrm{~min})$ que chez le rat $(23,5 \pm 3,3 \mathrm{~min}$, tableau I). Cependant, la surcharge hydrique induit une véritable diurèse aqueuse chez

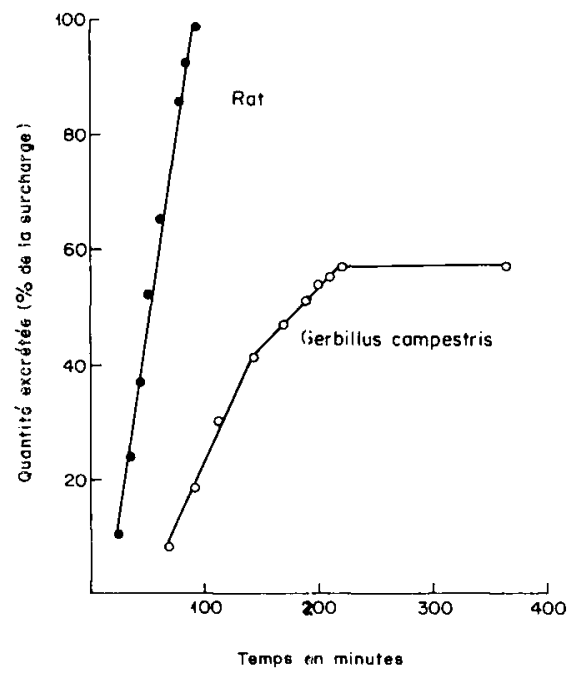

Fig 1. Cinétique d'excrétion urinaire d'une surcharge hydrique $(5 \%$ du poids corporel) chez le rat ( $\bullet$ ) et chez Gerbillus campestris (0). Chaque point représente la moyenne de 10 rats ou de 15 gerbilles.

la gerbille puisque la pression osmotique moyenne des urines, mesurée pour l'ensemble des gerbilles, reste nettement hypotonique par rapport au plasma pendant une durée relativement longue $(70 \mathrm{~min})$

Tableau I. Caractéristiques de la diurèse induite par une surcharge hydrique ( $5 \%$ du poids corporel) chez le rat et chez la gerbille. Chaque valeur représente la moyenne $\pm \operatorname{ESM}$ ( $n=$ nombre d'animaux). Les valeurs des pressions osmotiques correspondent aux moyennes des valeurs hypoosmotiques. La quantité excrétée correspond à la quantité totale d'urine récoltée pendant la durée de l'expérience $(7 \mathrm{~h})$.

\begin{tabular}{ccccc}
\hline Animal & $\begin{array}{l}\text { Délai } \\
(\mathrm{min})\end{array}$ & $\begin{array}{c}\text { Quantité excrétée } \\
(\% \text { surcharge })\end{array}$ & $\begin{array}{c}\text { Osmolalité urinaire } \\
\left(\mathrm{mOsm} . \mathrm{kg} \mathrm{H}_{2} \mathrm{O}^{-1}\right)\end{array}$ & $\begin{array}{c}\text { Quantité } \mathrm{H}_{2} \mathrm{O} \text { libre } \\
(\% \text { surcharge })\end{array}$
\end{tabular}

\begin{tabular}{lcccc}
\hline Rat & $23,5 \pm 3,3$ & $97,6 \pm 1,6$ & $42,0 \pm 2,9$ & $38,9 \pm 1,7$ \\
& $n=10$ & $n=10$ & $n=10$ & $n=10$ \\
Gerbille & $67,1 \pm 3,6$ & $57,5 \pm 2,7$ & $243 \pm 13$ & $14,2 \pm 1,9$ \\
& $n=15$ & $n=15$ & $n=15$ & $n=13$
\end{tabular}




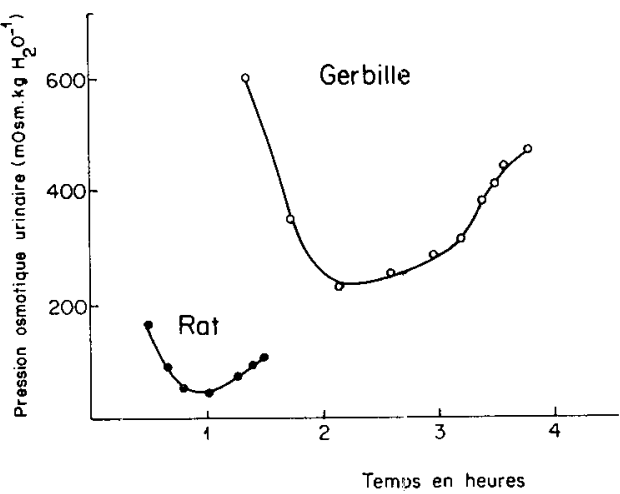

Fig 2. Évolution de la pression osmotique urinaire au cours de l'excrétion d'une surcharge hydrique ( $5 \%$ poids corporel) chez le rat et la gerbille. Chaque courbe représente la moyenne des résultats obtenus sur 10 rats (•) et 15 gerbilles (0). La pression osmotique au maximum de la diurèse est hypotonique au plasma chez tous les animaux étudiés.

(fig 2) et que la quantité d'eau libre excrétée (environ $0,18 \mathrm{ml}$, soit $14 \%$ de la surcharge, tableau I), bien que plus faible que celle du rat, n'est pas négligeable.

Tableau II. Osmolalité et ADH plasmatique chez les gerbilles recevant un régime alimentaire hydraté et des gerbilles en régime sec. Chaque valeur représente la moyenne \pm ESM ( $n=$ nombre d'animaux).

\begin{tabular}{lcc}
\hline Animaux & $\begin{array}{c}\text { Osmolalité } \\
\left(m \text { Osm॰kg } \mathrm{H}_{2} \mathrm{O}^{-1}\right)\end{array}$ & $\begin{array}{c}A D H \\
\left(p g \cdot m \digamma^{-1}\right)\end{array}$ \\
\hline Hydratés & $\begin{array}{c}302,27 \pm 2,66 \\
n=15\end{array}$ & $\begin{array}{c}164,37 \pm 13,36 \\
n=14\end{array}$ \\
Déshydratés & $\begin{array}{c}325,69 \pm 2,70 \\
n=29\end{array}$ & $\begin{array}{c}369,88 \pm 18,46 \\
n=24\end{array}$ \\
& $P<0,001$ & $P<0,001$ \\
\hline
\end{tabular}

\section{Modulation de la vasopressine au cours de la surcharge hydrique}

Les valeurs du tableau II montrent clairement que l'alimentation hydratée (apport de laitue) diminue significativement la concentration circulante de l'AVP. Les osmolalités plasmatiques sont corrélées significativement aux concentrations hormonales chez les animaux en régime sec $(r=0,92 ; P<0,001)$ et chez les animaux hydratés $(r=0,8 ; 0,001<P<0,01)$. La concentration de la vasopressine circulante au maximum de diurèse (quand les urines ont l'osmolalité la plus basse) est indétectable chez le rat, alors qu'elle reste élevée chez la gerbille $(55,0 \pm 6,7 \mathrm{pg} / \mathrm{ml})$ (tableau III). Ce rongeur désertique est donc capable d'élaborer une urine hypoosmotique par rapport au plasma pour des concentrations de vasopressine circulante relativement importantes.

\section{Effet de la dexaméthasone sur l'excrétion urinaire de Gerbillus campestris}

Dans ces conditions, l'excrétion de la surcharge est presque totale $(91,9 \pm 5,8 \%)$

Tableau III. Concentration plasmatique de l'hormone antidiurétique et osmolalité plasmatique au maximum de diurèse chez le rat et chez la gerbille. Chaque valeur représente la moyenne $\pm \operatorname{ESM}(n=$ nombre d'animaux).

\begin{tabular}{lcc} 
Animal & $\begin{array}{c}A D H \\
\left(p g \cdot \mathrm{m}^{-1}\right)\end{array}$ & $\begin{array}{c}\text { Osmolalité plasmatique } \\
\left(\mathrm{mOsm} . \mathrm{kg} \mathrm{H}_{2} \mathrm{O}^{-1}\right)\end{array}$ \\
\hline Rat & $\begin{array}{c}\text { Non détectable } \\
\text { Gerbille } \\
55,0 \pm 6,7 \\
n=10\end{array}$ & $\begin{array}{c}284,7 \pm 2,7 \\
304,5 \pm 2,5 \\
n=13\end{array}$ \\
\end{tabular}


(fig 3). Le délai d'installation de la diurèse est réduit de $13 \%$ chez les gerbilles traitées à la dexamethasone (tableau IV). Quand elles sont hypoosmotiques, les urines ont une pression osmotique moyenne de $100 \pm 5 \mathrm{mOsm} / \mathrm{kg} \mathrm{H}_{2} \mathrm{O}$ qui est significativement plus basse $(P<0,001)$ que celles des témoins (tableau IV).

\section{DISCUSSION}

La capacité d'excrétion d'une surcharge hydrique dépend du pouvoir de dilution du rein, de la fonction neurohypophysaire et de la fonction corticosurrénalienne. Le mécanisme de dilution, créé dans le segment large ascendant qui réabsorbe $\mathrm{NaCl}$ en excès sur l'eau, se poursuit dans les segments distaux et collecteurs qui, en l'absence d'ADH, sont peu perméables à l'eau et maintiennent la différence de pression osmotique transépithéliale établie par l'anse ascendante large (Bankir et al, 1989). Le

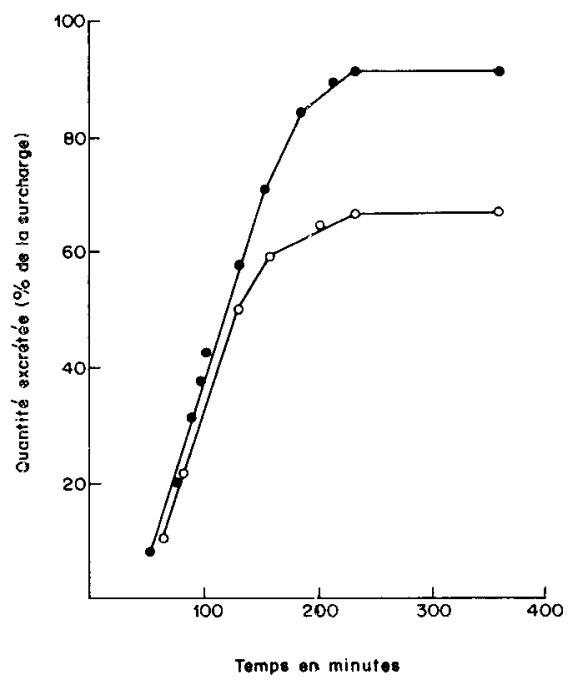

Fig 3. Cinétique d'excrétion urinaire d'une surcharge hydrique ( $5 \%$ du poids corporel) chez un même lot de Gerbillus campestris ayant reçu une injection de dexaméthasone (•) (15 $\mu \mathrm{g} /$ $100 \mathrm{~g}$ du poids corporel) ou de $\mathrm{NaCl} 0,9 \%(0)$. $n=8$ gerbilles.

Tableau IV. Caractéristique de la diurèse induite par une surcharge hydrique (5\% du poids corporel) chez la gerbille sans ou avec traitement à la dexaméthasone. La quantité excrétée d'urine totale au cours de l'expérimentation chez les gerbilles contrôles $(65,8 \pm 2,5 \%)$ n'est pas significativement différente de celle du lot I $(57,5 \pm 2,7 \%$, tableau I). Les valeurs des pressions osmotiques correspondent aux moyennes des pressions hypoosmotiques \pm ESM.

\begin{tabular}{lccc}
\hline Animaux & $\begin{array}{c}\text { Délai } \\
(\mathrm{min})\end{array}$ & $\begin{array}{c}\text { Quantité excrétée } \\
(\% \text { surcharge })\end{array}$ & $\begin{array}{c}\text { Osmolalité urinaire } \\
\left(\text { mOsm.kg H} \mathrm{O}^{-1}\right)\end{array}$ \\
\hline $\begin{array}{c}\text { Gerbilles contrôles } \\
n=8\end{array}$ & $62,9 \pm 3,8$ & $65,8 \pm 2,5$ & $135 \pm 6$ \\
$\begin{array}{c}\text { Gerbilles traitées } \\
\text { à la dexaméthasone } \\
n=8\end{array}$ & $55,1 \pm 5,7$ & $91,9 \pm 5,8$ & $100 \pm 5,0$ \\
& NS & $P<0,001$ & $P<0,001$ \\
\end{tabular}


travail de dilution du segment large ascendant dépend de la quantité de fluide tubulaire qu'il reçoit. En effet, si la filtration glomérulaire est basse, comme c'est le cas chez certains rongeurs désertiques (Baddouri et El Hilali, 1986), la faible quantité de fluide délivrée à l'anse de Henlé limitera la quantité d'eau libre excrétée par le rein. La capacité de dilution du rein dépend aussi de l'état d'hydratation de l'organisme au moment de la surcharge hydrique. Chez les rongeurs désertiques caractérisés par un important pouvoir de concentration du rein, la production d'urine hypotonique par administration d'une charge hydrique nécessite un conditionnement préalable pour abaisser le gradient osmotique du rein et réduire la réabsorption de l'eau qui reste non négligeable même en l'absence d'ADH (Bakir et de Rouffignac, 1985). Chez la gerbille, une alimentation riche en eau réduit significativement la concentration plasmatique de l'ADH qui passe d'environ 370 à $165 \mathrm{pg} / \mathrm{ml}$ en moyenne. Le prétraitement consistant à recevoir une charge en eau plusieurs jours avant l'expérience vise à réduire le gradient osmotique dans le rein et à limiter l'effet du stress dû au gavage intragastrique.

Comme chez la gerboise, Jaculus orientalis, une concentration relativement élevée de l'hormone antidiurétique dans le plasma de gerbille persiste pendant la diurèse (Baddouri et al, 1987) alors que l'hormone est indétectable au cours de la réponse diurétique chez le rat qui excrète la totalité de la surcharge. Ces résultats suggèrent que l'ADH à elle seule peut expliquer le pouvoir limité de dilution du rein de la gerbille. Il est probable que, malgré la surcharge d'eau chez cette espèce, la sécrétion de base de l'hormone reste élevée, ou que la vitesse d'inactivation de l'hormone reste lente. Une combinaison de ces 2 phénomènes peut toutefois exister.
L'amélioration de la diurèse sous l'effet du traitement à la dexaméthasone suppose qu'une concentration trop faible de glucocorticoïdes chez la gerbille pourrait être, au moins en partie, à l'origine du faible pouvoir de dilution du rein de ce rongeur. L'effet diurétique de la dexaméthasone, observé chez la gerbille, peut résulter soit d'un effet sur la filtration glomérulaire, soit d'une action freinatrice de ce stéroïde sur la sécrétion de l'ADH (Dingman et Despointes, 1960; MiahleVoloss et al, 1967). L'évaluation des glucocorticoïdes avant et au cours d'une charge d'eau permettrait de mieux apprécier le rôle de ces hormones dans l'excrétion de l'eau et éventuellement leurs contributions à l'économie de l'eau chez ces espèces désertiques.

\section{RÉFÉRENCES}

Ahmed ABJ, George C, Gonzales-Auvert C, Dingman If (1967) Increased plasma arginine-vasopressin in clinical adrenocortical insufficiency and its inhibition by glucosteroids. $J$ Clin Invest 46, 111-123

Baddouri KH, El Hilali M (1986) Sécrétion de I'hormone antidiurétique et fonction rénale au cours du réveil de l'hibernation de Jaculus orientalis. J Physiol 81, 202-208

Baddouri KH, El Hilali M, Marchetti J, Menard J (1987) Renal excretion capacity in hydrated desert rodents (Jaculus orientalis and Jaculus deserti). J Comp Physiol 157, 237-240

Bankir L, De Rouffignac C (1985) Urinary concentrating ability: Insights from comparative anatomy. Am J Physiol 249 (Regul integr Comp Physiol 18) R643-R666

Bankir L, Bouby N, Trinh-Trang-Tan MM (1989) The role of the kidney in the maintenance of water balance. Water and salt homeostasis in health and disease. Clin Endocrinol Métab 3, 249-311

Boykin J, Detorrente A, Erikson A, Robertson G, Schrier RW (1978) Role of plasma vasopres$\sin$ in impaired water excretion of glucocorticoid deficiency. J Clin Invest 62, 738-744 
Dingman JF, Despointes RH (1960) Adrenal steroid inhibition of vasopressin release from the neurohypophysis of normal subjects and patients with Addison's disease. J Clin Invest 39, 1851-1863

Green NH, Harringhton AR, Valtin H (1970) On the role of antidiurectic hormone in inhibition of acute water diuresis in adrenal insufficiency and the effects of gluco- and mineralocorticoid in reversing the inhibition. $J$ Clin Invest 49, 1724-1736

Hofman FG (1956) Control of water and salt electrolyte metabolism in vertebrates (C Jone, P Eckstein, eds). Cambridge University Press, 38-40

Hummel VR (1963) The physiological adaptations of desert rodents. In: Rodents in desert environment (Prakash, Ghosh, eds) ch XXI, 413-444

Kleeman CR, Czaczkes JW, Cuttler R (1964) Mechanisms of impaired water excretion in adrenal and pituitary insufficiency IV. Antidiuretic hormone in primary and secondary adrenal insufficiency. $J$ Clin Invest 43,1641 1648
Klein LA, Roth J, Petersen MJ (1966) Radioimmunoassay of arginine vasopressin. Surg Forum 17, 240

Linas SL, Berl T, Robertson GL, Aisenbrey GA, Schrier RW (1980) Role of plasma vasopres$\sin$ in the impaired water excretion of glucocorticoid deficiency. Kidney int 18, 58-67

Mandel IN, De Fronzo RA, Robertson GL, Forrest JNJRO (1980) Role of plasma arginine vasopressin in the impaired water diuresis of isolated glucocorticoid deficiency in the rat. Kidney Int 17, 186-195

Marchetti J (1973) Immunoassay for lysinevasopressin (LVP): comparison of biological and immunological activity of lysinevasopressin and some of its synthetic analogues. Experientia 29, 3, 351-353

Miahle-Voloss C, Lutz B, Koch B (1967) Effet inhibiteur de la dexamethasone sur la sécrétion de la vasopressine et dosage de l'ADH. CR Acad Sci Paris 264, 2145-2147

Petter F (1961) Répartition géographique et écologie des rongeurs désertiques. Mammalia 25 ( ${ }^{\circ}$ spécial) 